\title{
Instabilities in two-liquid layers subject to a horizontal temperature gradient*
}

\author{
S. Madruga ${ }^{1}$, C. Pérez-García ${ }^{2,3}$, G. Lebon ${ }^{1}$ \\ 1 Departement AGO, Liège University, B5, Sart-Tilman, 4000 Liège, Belgium \\ ${ }^{2}$ Instituto de Física, Universidad de Navarra, 31080 Pamplona, Spain \\ ${ }^{3}$ Depto. Ingen. Mecánica, E. T. S. Ingenieros (TECNUN), Univ. Navarra, 20018 San Sebastián, Spain
}

\begin{abstract}
We study theoretically the stability of two superposed fluid layers heated laterally. The fluids are supposed to be immiscible, the interface undeformable and of infinite horizontal extension. Combined thermocapillary and buoyancy forces give rise to a basic flow when a temperature difference is applied. The calculations are performed for a melt of GaAs under a layer of molten $\mathrm{B}_{2} \mathrm{O}_{3}$, a configuration of considerable technological importance. Four different flow patterns and five temperature configurations are found for the basic state in this system. A linear stability analysis shows that the basic state may be destabilized by oscillatory motions leading to the so-called hydrothermal waves. Depending on the relative height of the two layers these hydrothermal waves propagate parallel or perpendicular to the temperature gradient. This analysis reveals that these perturbations can alter significantly the liquid flow in the liquid-encapsulated crystal growth techniques.
\end{abstract}

Key words: convection, thermocapillary flow, hydrodynamic instability

PACS: 47.20.Dz, 47.20.Bp, 47.54.+r, 47.27.Te, 44.25.+f, 47.20.Ma

\section{Introduction}

In recent years, convective flows in systems with free boundaries have attracted much attention, mainly owing to their relevance in many processes of technological interest. When the temperature at the interface between two immiscible fluids is not uniform, a flow may be induced due to the temperature dependence of surface tension. This flow is usually called thermocapillary convection or Marangoni convection and can arise in a liquid-gas or a liquid-liquid interface subjected to a temperature gradient. The simplest convective flow appears when a free surface of an single extended liquid layer is subject to a horizontal temperature gradient. As soon as a lateral heating is applied a basic flow settles down in the liquid layer and the resulting temperature profile is highly nonlinear. This flow destabilizes through an oscillatory instability that induces wave motions in simple fluids called hydrothermal waves (Smith and Davis [14]).

Correspondence to: C. Pérez-García (e-mail: carlos@ isicica.unav.es)

* This work has been supported by the contracts ICOPAC 2000-00136 (European Union), ESA CIMEX, DGICYT (Spanish Government) grant BFM2002-O1002, PIUNA (Univ. Navarra) and the "Asociación de Amigos" (Univ. Navarra). 
Davis and Smith [14] showed that the instability mechanism even in this simple case is quite complex, resulting from the interplay between the basic flow and thermal or velocity disturbances. At low Prandtl numbers $(P r)$, hydrothermal waves propagate in a direction perpendicular to the horizontal temperature gradient, while at high $P r$, they advance parallel to the temperature gradient. At intermediate $P r$, the waves form an angle with the streamwise direction (Smith [13]). Later on, some authors (Parmentier et al. [8], Mercier and Normand [6]) extended these calculations by taking into account buoyancy effects and thermal transfer properties at the interface. Daviaud and Vince [3] reported the first observation of hydrothermal waves in a shallow layer of $0.65 \mathrm{cSt}$ silicon oil. These observations were complemented by other authors considering different liquids and geometries (Mukolobwiez et al. [7], Riley and Neitzel [12], Priede and Gerbeth [11], Pelacho et al. [9], Burguete et al. [1]).

A common simplification in dealing with thermocapillary flows consists of considering convection solely in the liquid layer, while the effects of the upper gas are taken into account through the Biot number, an empirical parameter characterizing the conductive heat transfer across interfaces. To justify this approximation or in dealing with a liquid-liquid interface, a full hydrodynamics analysis of the two-layer system is required. Thermocapillary flows in several pairs of liquids have been considered in a previous paper (Madruga et al. [5]). Here we study the behaviour of $\mathrm{GaAs}-\mathrm{B}_{2} \mathrm{O}_{3}$ because of its practical interest in liquid encapsulation technique used to minimize evaporation of the volatile component (such as arsenic in GaAs) from the melt during the crystal growth of some materials (Campbell and Kostner [2]). This technique consists in encapsulating the melt with a low melting point amorphous molten glass phase, such as boron oxide $\left(\mathrm{B}_{2} \mathrm{O}_{3}\right)$ (Prakash and Kostner [10]). Often it is technically important therefore, to study the dynamics of this two-layer system to avoid oscillatory flows which may result into undesired effects on the crystal properties (Liu et al. [4]). and to confirm that that the main conclusions remain valid, whatever the diversity of the velocity and temperature profiles and the nature of the various fluid.

The purpose of this study is to investigate theoretically the main flow regimes in a system of two molten phases ( $\mathrm{GaAs}$ and $\mathrm{B}_{2} \mathrm{O}_{3}$ ) subjected to lateral heating. We perform a linear stability analysis to determine the convective instabilities and the characteristics of hydrothermal waves in the same two-layer system. In Sect. 2 we formulate the problem and discuss the governing equations and boundary conditions. Velocity and temperature profiles of the basic flow are discussed in Sect. 3. Section 4 contains the linear stability analysis of this flow and the main results on hydrothermal waves. Finally, we discuss the consequences of this study with respect to the encapsulating technique in Sect. 5.

\section{Governing equations}

We consider a system of two horizontal superimposed immiscible liquid layers of thickness $h^{(i)}$, density $\rho^{(i)}$, kinematic viscosity $v^{(i)}$, coefficient of thermal expansion $\alpha^{(i)}$, thermal conductivity $\lambda^{(i)}$ and thermal diffusivity $\kappa^{(i)}$. Superscript $i=1,2$ refers to the lower and upper fluid, respectively. The system is infinite in the horizontal extent and is limited in the vertical direction by two horizontal rigid walls (see Fig. 1). The interface between the two liquids is assumed to be flat and nondeformable. The origin of the Cartesian reference system is fixed at the interface.

The system is heated, with a constant temperature gradient $\beta$ imposed along the horizontal direction, which produces a conducting temperature profile given by

$$
T=T_{-}+\beta x,
$$

where $T_{-}$is the temperature of the cold side. The system is subject to the gravity field and its density is given by the state equation $\rho^{(i)}=\rho_{0}^{(i)}\left[1-\alpha^{(i)}\left(T-T_{-}\right)\right]$. The interfacial tension depends on temperature variations through the relationship $\sigma=\sigma_{0}-\gamma\left(T-T_{-}\right)$where $\gamma=-\frac{\partial \sigma}{\partial T}$ is a positive constant (at least for usual liquids). The Boussinesq approximation is taken for granted in both layers. The governing equations expressing the balance of mass, momentum, and energy under this approximation using nondimensional variables are given 
Instabilities in two-liquid layers subject to a horizontal temperature gradient

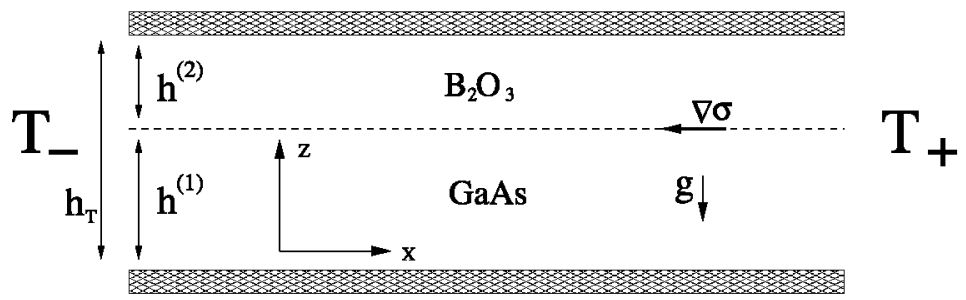

Fig. 1. Diagram of the two-layer system. Two immiscible liquids superposed are subjected to a horizontal gradient of temperature. The forces acting on the system are gravity and interfacial tension

by (Madruga et al. [5])

$$
\begin{aligned}
\nabla \cdot \mathbf{u}^{(i)} & =0, \\
\operatorname{Pr}^{-1}\left[\partial_{t} \mathbf{u}^{(i)}+\left(\mathbf{u}^{(i)} \cdot \nabla\right) \mathbf{u}^{(i)}\right] & =-\frac{1}{\rho^{*}} \nabla p^{(i)}-\left[\frac{\left(h^{(1)}\right)^{3} g}{v^{(1)} \kappa^{(1)}}-\alpha^{*} R a\left(T^{(i)}-T_{-}\right)\right] \hat{z}+v^{*} \nabla^{2} \mathbf{u}^{(i)}, \\
\partial_{t} T^{(i)}+\left(\mathbf{u}^{(i)} \cdot \nabla\right) T^{(i)} & =\kappa^{*} \nabla^{i} T^{(i)} .
\end{aligned}
$$

wherein $\boldsymbol{u}^{(i)}\left(\boldsymbol{u}^{(i)}, v^{(i)}, w^{(i)}\right)$ is the velocity field, $p^{(i)}$ the pressure, and $\hat{z}$ the unit vector in the vertical direction and in which the following scales: $h^{(1)}$ length, $h^{(1)^{2}} / \kappa^{(1)}$ time, $\kappa^{(1)} / h^{(1)}$ velocity, $\rho^{(1)} v^{(1)} \kappa^{(1)} / h^{(1)^{2}}$ pressure, and $\beta h^{(1)}$ temperature have been used. Coefficients with a hat ${ }^{\wedge}$ take the values: a) 1 in fluid (1), and b) the ratios $\alpha^{*}=\alpha^{(2)} / \alpha^{(1)}, \kappa^{*}=\kappa^{(2)} / \kappa^{(1)}, v^{*}=v^{(2)} / \nu^{(1)}, \lambda^{*}=\lambda^{(2)} / \lambda^{(1)}, \rho^{*}=\rho^{(2)} / \rho^{(1)}$, $\mu^{*}=\rho^{(2)} v^{(2)} / \rho^{(1)} v^{(1)}=\rho * \nu *$, and $a=h^{(2)} / h^{(1)}$ in fluid (2). The nondimensional boundary conditions are (Madruga et al. [5]):

$$
\begin{aligned}
& \mathbf{u}^{(i)}=0, \quad T^{(i)}=T_{w}\left(T_{w}=T_{-}+x\right) \text { at } z=-1, a \\
& \mathbf{u}^{(1)}=\mathbf{u}^{(2)}, \quad w^{(1)}=w^{(2)}=0, \quad T^{(1)}=T^{(2)}, \\
& \partial_{z} T^{(1)}=\lambda^{*} \partial_{z} T^{(2)}, \quad \mu^{*} \partial_{z} \mathbf{u}_{h}^{(2)}-\partial_{z} \mathbf{u}_{h}^{(1)}=M a \nabla_{h} T^{(1)} \text { at } z=0
\end{aligned}
$$

The nondimensional numbers, i.e., the Prandtl number $\operatorname{Pr}=v^{(1)} / \kappa^{(1)}$, the Rayleigh number $R a=$ $\frac{\alpha^{(1)} \beta g\left(h^{(1)}\right)^{4}}{\nu^{(1)} \kappa^{(1)}}$ ( $g$ the acceleration of gravity), and the Marangoni number $M a=-\frac{\mathrm{d} \sigma}{\mathrm{d} T} \frac{\beta\left(h^{(1)}\right)^{2}}{\rho^{(1)} \nu^{(1)} \kappa^{(1)}}$, are defined with respect to liquid 1 . In the general case of a partially conducting walls the thermal boundary condition takes the form $\partial T^{(i)}=-B i\left(T^{(i)}-T_{w}\right)$, where $B i$ indicates the Biot number that accounts for heat exchanges through interfaces.

\section{Basic state}

When a horizontal temperature gradient is applied a stationary basic state of the form $T^{(i)}=T_{-}+x+\tau^{(i)}(z)$, $\mathbf{u}_{b}^{(i)}=\left(u_{0}^{(i)}(z), 0,0\right)$ sets in. In order to determine this state, the return flow condition must be applied (Smith and Davis [14]). This requires that the net flow through a vertical plane must vanish in each layer, i.e.,

$$
\int_{-1}^{0} u_{0}^{(1)}=\int_{0}^{a} u_{0}^{(2)}=0,
$$

These conditions, together with the governing equations and boundary conditions determine the velocity and temperature fields $u_{0}^{(i)}$ and $\tau^{(i)}$ of the basic flow. Explicit expressions of these fields are easily obtained with Maple or Mathematica. In the lower liquid (1) and for conducting upper and lower plates these fields take the 
Table 1. Physical parameters of $\mathrm{B}_{2} \mathrm{O}_{3}$ and $\mathrm{GaAs}$ (Prakash and Koster [10])

\begin{tabular}{llllllll}
\hline Liquid & $\rho\left(\mathrm{kg} / \mathrm{m}^{3}\right)$ & $v\left(\mathrm{~m}^{2} / \mathrm{s}\right)$ & $\lambda(\mathrm{J} / \mathrm{msK})$ & $c_{p}(\mathrm{~J} / \mathrm{kgK})$ & $\alpha\left(\mathrm{K}^{-1}\right)$ & $\gamma(\mathrm{N} / \mathrm{mK})$ & $\operatorname{Pr}$ \\
\hline $\mathrm{B}_{2} \mathrm{O}_{3}$ & 1648 & $2370 \cdot 10^{6}$ & 2 & 481.58 & $0.09 \cdot 10^{-3}$ & 939.1 \\
$\mathrm{GaAs}$ & 5720 & 0.49 & 17.8 & 434.01 & 0.187 & 0.068 \\
$\mathrm{~B}_{2} \mathrm{O}_{3} / \mathrm{GaAs}$ & $\rho^{*}=0.288$ & $v^{*}=4836.73$ & $\lambda^{*}=0.112$ & $c_{p}^{*}=c_{p}^{(2)} / c_{p}^{(1)}=1.1$ & $\alpha^{*}=481$ & $-1.2 \cdot 10^{-3}$ \\
\hline
\end{tabular}

form:

$$
\begin{aligned}
u_{0}^{(1)}= & \frac{1}{48\left(a+\mu^{*}\right) v^{*}}\left[-12 a M a v^{*}\left(1+4 z+3 z^{2}\right) .\right. \\
& +R a\left\{(a)^{3} \alpha^{*} \mu^{*}-a v^{*}+4 \mu^{*}\left((a)^{3} \alpha^{*}+v^{*}\right) z+3\left((a)^{3} \alpha^{*} \mu^{*}+3 a v^{*}+4 \mu^{*} v^{*}\right) z^{2}\right. \\
& \left.\left.+8\left(a+\mu^{*}\right) v z^{3}\right\}\right] \\
\tau^{(1)}= & \frac{1}{2880\left(a+\lambda^{*}\right)\left(a+\eta^{*}\right) v^{*} \kappa^{*}}[ \\
& 60 M a\left\{(a)^{2} v^{*}\left(h^{*} \lambda^{*}+\kappa^{*}\right)+a \lambda^{*} v^{*}\left((a)^{2}-\kappa^{*}\right) z-6 a\left(h^{*}+\lambda^{*}\right) v^{*} \kappa^{*} z^{2}\right. \\
& \left.-8 a\left(a+\lambda^{*}\right) v^{*} \kappa^{*} z^{3}-3 a\left(a+\lambda^{*}\right) v^{*} \kappa^{*} z^{4}\right\} \\
& +\operatorname{Ra}\left\{a v^{*}\left(5(a)^{2} \lambda^{*}+9 a \kappa^{*}+4 \eta^{*} \kappa^{*}\right)-(a)^{4} \alpha^{*}\left(4\left(h^{*}\right)^{2} \lambda^{*}+9 a \lambda^{*} \eta^{*}+5 \eta^{*} \kappa^{*}\right)\right. \\
& -\left(v^{*}\left(-5(a)^{3}+9 a \kappa^{*}+4 \eta^{*} \kappa^{*}\right)+\alpha^{*}\left(4\left(h^{*}\right)^{6}+9(a)^{5} \eta^{*}-5(a)^{3} \eta^{*} \kappa^{*}\right)\right) \lambda^{*} z \\
& +30 a\left(a+\lambda^{*}\right)\left((a)^{2} \alpha^{*} \eta^{*}-v^{*}\right) \kappa^{*} z^{2}+40\left(a+\lambda^{*}\right) \eta^{*}\left((a)^{3} \alpha^{*}+v^{*}\right) \kappa^{*} z^{3} \\
& \left.\left.+15\left(a+\lambda^{*}\right)\left(\left(h^{*}\right)^{3} \alpha^{*} \eta^{*}+3 a v^{*}+4 \eta^{*} v^{*}\right) \kappa^{*} z^{4}+24\left(h^{*}+\lambda^{*}\right)\left(a+\eta^{*}\right) v^{*} \kappa^{*} z^{5}\right\}\right]
\end{aligned}
$$

and similar expressions for fluid (2) (see appendix in (Madruga et al. [5])). The important point here is that the velocity field is typically a polynomial of order three and the temperature field of order five in $z$.

Because of the great number of parameters involved in the calculation of $\tau^{(i)}(z)$ and $u^{(i)}(z)$ the basic state will be discussed for a specific configuration. We chose a melt of $G a A s$ (lower liquid) under a layer of molten $\mathrm{B}_{2} \mathrm{O}_{3}$ (upper liquid), because of its technological interest. (Other cases have been treated in (Madruga et al. [5])). The physical properties of these materials are listed in Table 1 (Prakash and Koster [10]).

The thermal conductivities of these two fluids differ by a factor of 10 . So, even if we assume that the top and bottom plates are made of the same material, the resulting boundary condition should differ by the same factor. As a consequence we assume a finite $B i$ in these two rigid plates: $B i^{(1)}=0.1$ and $B i^{(2)}=1$. Apart from $R a$ and $M a$, which depend on the temperature gradient $\beta$, there are two additional control parameters: the total depth $h_{T}$ and the depth ratio between the liquids, taken as the percentage of the bottom depth ratio

$$
\hat{h}_{1}=100 /(1+a) .
$$

In a diagram with these two parameters as axes, four velocity profiles (see Fig. 2) and five regions for the temperature field are distinguished (see Fig. 3). The interaction between interfacial tension and buoyancy gives rise to the following configurations: 1) a counter-rotating cell in each layer, 2) two counter-rotating cells in the upper liquid and one cell in the lower one, in both cases with a flow near the interface from hot to cold, 3) two counter-rotating cells in the lower liquid and one cell in the upper one, 4) a counterrotating cell in each liquid, but with the flow being dragged near the interface from cold to hot in the last two cases.

We have also determined the different stratified temperature profiles that result from the interplay between thermal diffusivity and the fluid flow. The possible temperature profiles for the lower fluid include: unstably stratified (temperature increases with depth), stably stratified (temperature decreases with depth), and inverse $S$-shaped profiles. The upper liquid does not display a stably stratified profile, but it may exhibit a $S$-shaped one. An increase in the conductivity of the walls affects mainly region (I) which is shifted towards higher $\hat{h}_{1}$ values and eventually may disappear. 


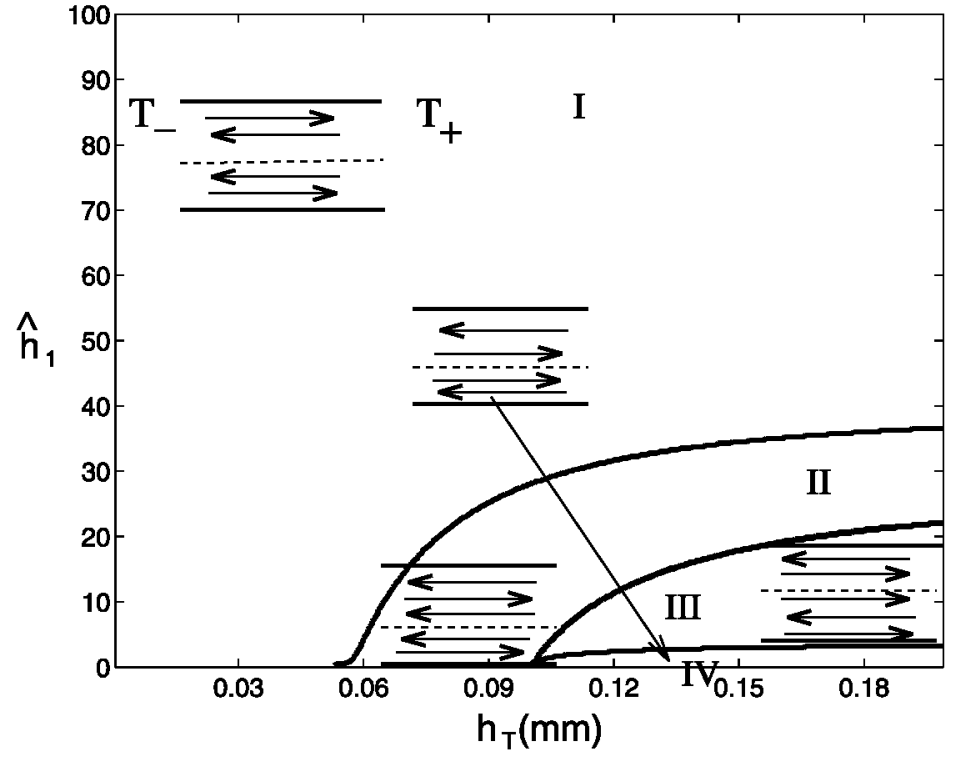

Fig. 2. Velocity profiles of the basic state for a system composed by a melt of GaAs (lower liquid) and a layer of molten $\mathrm{B}_{2} \mathrm{O}_{3}$ (upper liquid). The Biot numbers at the lower and upper wall are taken equal to $B i^{(1)}=0.1$ and $B i^{(2)}=1$, respectively

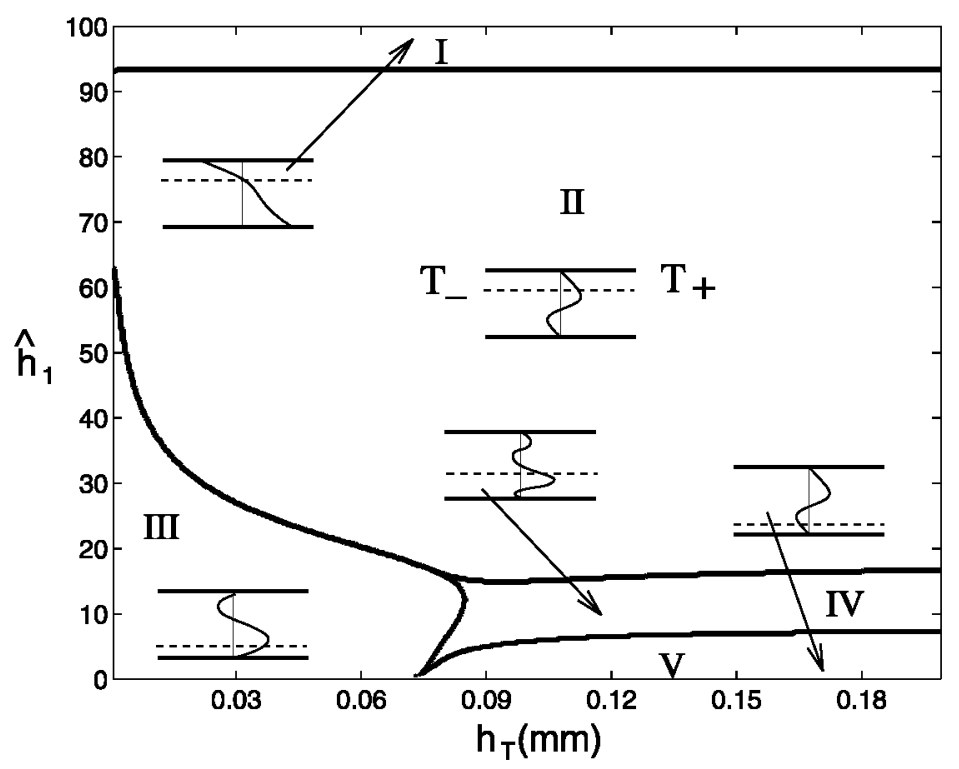

Fig. 3. Temperature profiles of the basic state

\section{Stability analysis}

In a linear stability analysis small perturbations around the basic flow

$$
\mathbf{u}^{(i)}=\left(u_{0}^{(i)}+u^{\prime(i)}, v^{(i)}, w^{\prime(i)}\right), \quad T^{(i)}-T_{-}=x+\tau^{(i)}+\theta^{\prime(i)}, \quad p^{(i)}=p_{0}^{(i)}+p^{(i)}
$$

are considered, where the superscript ' denotes the perturbations of velocity, temperature, and pressure fields, respectively. In turn, these perturbations are expanded in normal modes

$$
\left(\mathbf{u}^{\prime}, \theta^{\prime}, p^{\prime}\right)=[\overline{\mathbf{u}}(z), \bar{\theta}(z), \bar{p}(z)] \exp i(\mathbf{k} \cdot \mathbf{x}-\omega t) .
$$

Here $\omega$ denotes the frequency, $k_{x}$ is the wavenumber in the streamwise direction and $k_{y}$ the wavenumber in the spanwise direction. Eliminating the pressure, the equations for the perturbations $(u, w, \theta)$ (the bars are 

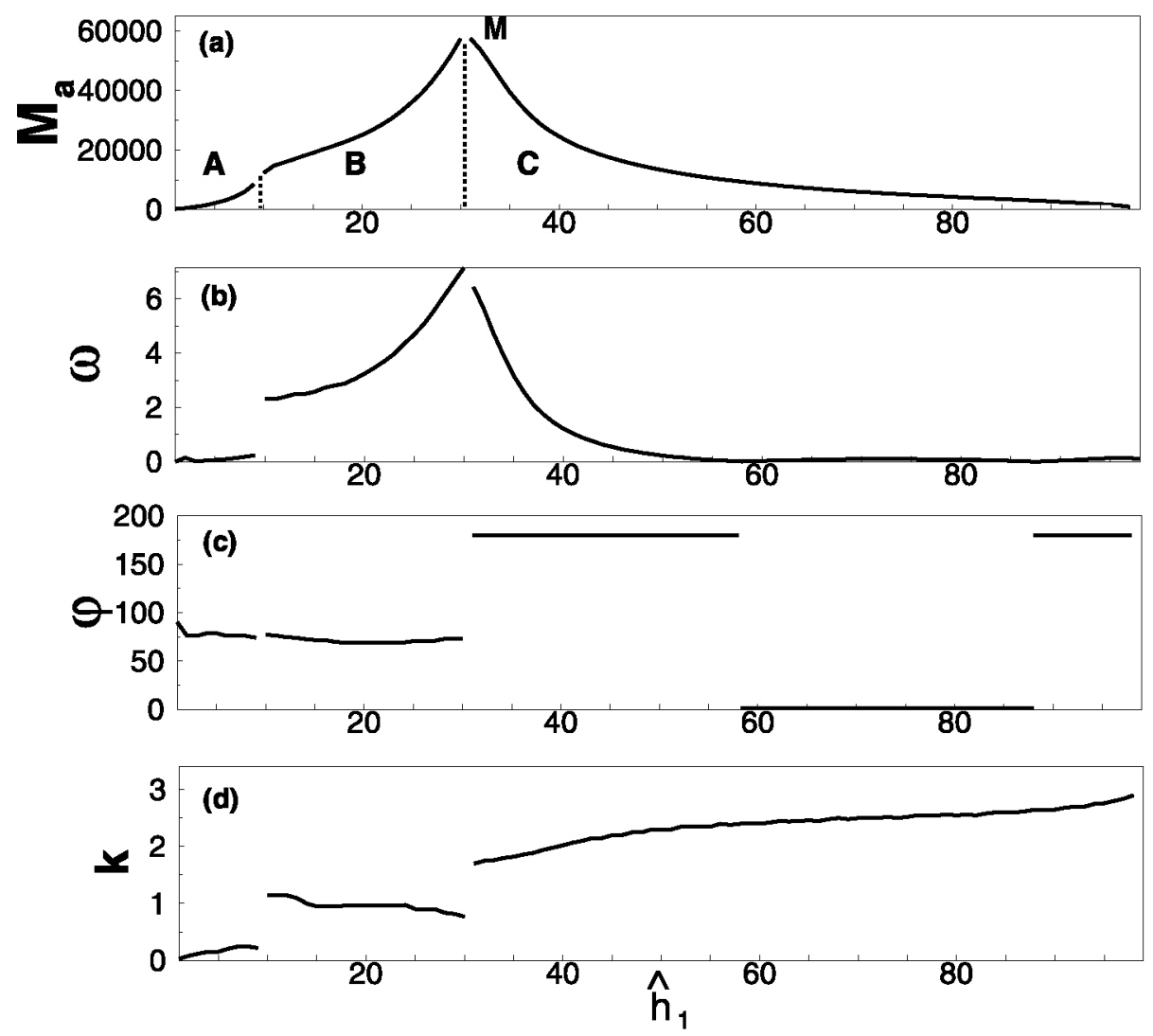

Fig. 4a-d. Critical parameters for the system $\mathrm{B}_{2} \mathrm{O}_{3}$ (upper liquid) and $\mathrm{GaAs}$ (lower liquid) with total depth $6 \mathrm{~mm}$. (a) Marangoni, (b) frequency, (c) angle of the wavector, (d) modulus of the wavevector

dropped for clarity) read as:

$$
\begin{aligned}
& v^{*} \partial_{z}^{2} u^{(i)}-L^{(i)} u^{(i)}-\frac{k_{y}^{2}}{k^{2}} \operatorname{Pr}^{-1} \partial_{z} u_{0}^{(i)} w^{(i)}-i \frac{k_{x}}{k^{2}}\left(v^{*} \partial_{z}^{3} w^{(i)}-L^{(i)} \partial_{z} w^{(i)}\right)=0 \\
& v^{*} \partial_{z}^{4} w^{(i)}-\left(v^{*} k^{2}+L^{(i)}\right) \partial_{z}^{2} w^{(i)}+k^{2} L^{(i)} w^{(i)}-R a k^{2} \alpha^{*} \theta^{(i)}+i k_{x} \operatorname{Pr}^{-1} w^{(i)} \partial_{z}^{2} u_{0}^{(i)}=0 \\
& \kappa^{*} \partial_{z}^{2} \theta^{(i)}-\left(\kappa^{*} k^{2}-i\left(\omega+u_{0}^{(i)} k_{x}\right)\right) \theta^{(i)}-u^{(i)}+\partial_{z} \tau^{(i)} w^{(i)}=0
\end{aligned}
$$

in which linear operator $L^{(i)}=v^{*} k^{2}+i \operatorname{Pr}^{-1}\left(\omega+u_{0}^{(i)} k_{x}\right)$ has been introduced, with the ensuing boundary conditions

$$
\begin{aligned}
& u^{(i)}=w^{(i)}=\partial_{z} w^{(i)}=\partial_{z} \theta^{(i)}+B i^{(i)} \theta^{(i)}=0 \quad \text { at } z=-1, a \\
& u^{(1)}=u^{(2)}, \quad \partial_{z} w^{(1)}=\partial_{z} w^{(2)}, w^{(1)}=w^{(2)}=0 \\
& \theta^{(1)}=\theta^{(2)}, \partial_{z} \theta^{(1)}=\lambda^{*} \partial_{z} \theta^{(2)}, \mu^{*} \partial_{z} u^{(2)}-\partial_{z} u^{(1)}=i k_{x} M a \theta^{(1)} \\
& \mu^{*} \partial_{z}^{2} w^{(2)}-\partial_{z}^{2} w^{(1)}=\operatorname{Mak}^{2} \theta^{(1)} \text { at } z=0 .
\end{aligned}
$$

This set of equations is solved by a tau-Chebyshev spectral method in which the eigenfunctions are approximated by Chebyshev polynomials up to 16th order to ensure a good convergence of the eigenvalues. The marginal curves are found by searching the set of values $k_{x}, k_{y}, M a$ which correspond to a vanishing real part of the temporal growth rate.

In Fig. 4 are represented the critical Marangoni number $M a$, the frequency of the waves $\omega$, the angle of propagation of the waves $\varphi$ with respect to the temperature gradient, and the wavenumber $k$, as a function of the percentage of the bottom depth ratio $\hat{h}_{1}$ for the pair of liquids $\mathrm{B}_{2} \mathrm{O}_{3} / \mathrm{GaAs}$ and the total depth $h_{T}=6 \mathrm{~mm}$. The system can be destabilized by several mechanisms: unstably stratified sublayers giving 
rise to a Rayleigh-Bénard instability, Marangoni instability at the interface, or the horizontal velocity at the interface overcoming the stabilizing effect of a given vertical temperature field. For a wide range around $\hat{h}_{1}=30 \%$ (see point $M$ in Fig. 4 a) the system is strongly stabilized. This means that a combination of $\hat{h}_{1}=1 / 3 h_{T}(\mathrm{GaAs})$ and $\hat{h}_{2}=2 / 3 h_{T}\left(\mathrm{~B}_{2} \mathrm{O}_{3}\right)$ is particularly suitable in preventing oscillatory motions. The position of this maximum point depends on the properties of the pair of liquid and the total depth considered. In general point M shifts to lower values of $\hat{h}_{1}$ when $v^{*}$ or $\kappa^{*}$ increase, while it tends to appear at greater values of $\hat{h}_{1}$ when $h_{T}$ is increased (Madruga et al. [5]). Notice that the value $v^{*}=4836.73$ for $\mathrm{GaAs}-\mathrm{B}_{2} \mathrm{O}_{3}$ is much greater than the values $v^{*}=1-10$ for the pairs of liquids considered in Madruga et al. [5]. The point $M$ corresponds to a codimension-two point resulting from the interaction of two Hopf modes with different wavenumbers and propagating in opposite directions (see Figs. 4c, 4d).

In this region, the $\mathrm{GaAs}$ component has a stable temperature profile, but $\mathrm{B}_{2} \mathrm{O}_{3}$ exhibits a temperature profile with three sublayers: a middle unstably stratified region, and two thermally stable zones, one in contact with the upper wall and another including the interface. The flows have different characteristics in the regions denoted $\mathrm{A}, \mathrm{B}$ and $\mathrm{C}$ in Fig. $4 \mathrm{a}$. In regions $\mathrm{A}$ and $\mathrm{B}$, hydrothermal waves propagate with $\varphi$ close to $90^{\circ}$. The difference between regions (A) and (B) is mainly due to the modulus and frequency of the critical modes, being both smaller in (A). In region (C), the hydrothermal waves propagate from hot to cold for $31 \% \leq \hat{h}_{1} \leq 58 \%$ and $88 \% \leq \hat{h}_{1} \leq 100 \%$, and from cold to hot when $59 \% \leq \hat{h}_{1} \leq 87 \%$. However for $\hat{h}_{1}>58 \%$ the frequency of the waves is very small (about $O\left(10^{-2}\right)$ ) and therefore they will seen as stationary transverse rolls in an experiment. The dynamics in this region is clearly dominated by the lower liquid. Let us mention that these stability results agree qualitatively with the results in other pairs of fluids, although the stability thresholds depend strongly on the properties of the particular fluids studied (Madruga et al. [5]).

\section{Conclusions}

We studied the stability of two superposed, immiscible fluid layers heated with a horizontal thermal gradient. The lateral heating gives rise to a global circulation (basic flow) in each layer. Depending on the interaction between interfacial tension and buoyancy the following flows are obtained: 1) a counter-rotating cell in each layer, 2) two counter-rotating cells in the upper liquid and one cell in the lower one (in both cases one observes a flow from hot to cold near the interface), 3) to two counter-rotating cells in the lower liquid and a cell in the upper one, and 4) a counter-rotating cell in each liquid, but with the flow being dragged near the interface from cold to hot in the last two cases. We have also determined the different temperature profiles that result from the interplay between thermal diffusivity and fluid motion. These basic flows can become unstable through three different patterns: 1) hydrothermal waves propagating from cold to hot side with a small angle, which is typical of lateral heating in one-liquid systems with high $\operatorname{Pr}, 2$ ) hydrothermal waves propagating from hot to cold, and 3 ) longitudinal stationary rolls.

Although these results were calculated for a system of infinite horizontal extend, with approximated Biot numbers, and for a particular pair of molten phases ( $\mathrm{GaAs}$ encapsulated with $\mathrm{B}_{2} \mathrm{O}_{3}$ ) they confirm that hydrothermal waves may be a source of inconvenient disturbances while using the encapsulating technique to grow electronic materials. Moreover the main conclusions remain valid, whatever the diversity of the velocity and temperature profiles and the nature of the various fluids (Madruga et al. [5]). As a consequence any numerical code intending to reproduce an encapsulating device or a two-fluid layer has to consider stability against transverse hydrothermal waves.

Acknowledgments. The authors wish to thank M. Dondlinger and T. Desaive (Liège), H. Mancini, J. Bragard, and J. Burguete (Pamplona) for helpful discussions and comments.

\section{References}

1. Burguete, J., Mukolobwiez, N., Daviaud, F., Garnier, N., Chiffaudel, A.: Buoyant-thermocapillary instabilities in an extended liquid layer subjected to a horizonal temperature gradient. Phys. Fluids 13, 2773-2787 (2001)

2. Campbell, T.A., Koster, J.: Modelling of liquid encapsulated gallium melts. Acta Astronaut. 35, 805-812 (1995) 
3. Daviaud, F., Vince, J.: Traveling waves in a fluid layer subjected to a horizontal temperature gradient. Phys. Rev. E 48,4432 (1993)

4. Liu, Q.S., Roux, B., Velarde, M.G.: Thermocapillary convection in two-layer systems. Int. J. Heat Mass Transfer 41, 14991511 (1998)

5. Madruga, S., Pérez-García, C., Lebon, G.: Convective instabilities in two superposed horizontal liquid layers heated laterally. Phys. Rev. E 68, 041607 (2003)

6. Mercier, J., Normand, C.: Buoyant thermocapillary instabilities of differentially heated liquid layers. Phys. Fluids 8, 14331445 (1996)

7. Mukolobwiez, N., Chiffaudel, A., Daviaud, F.: Supercritical Eckhaus instability for surface-tension-driven hydrothermal waves. Phys. Rev. Lett. 80, 4661 (1998)

8. Parmentier, P.M., Regnier, V., Lebon, G.: Bouyant-thermocapillary instabilities in medium $P_{r}$ number fluid layers subject to a horizontal temperature gradient. Int. J. Heat Mass Trans. 36, 2417-2427 (1993)

9. Pelacho, M.A., Garcimartín, A., Burguete, J.: Local Marangoni number at the onset of hydrothermal waves. Phys. Rev. E 62, 477-483 (2000)

10. Prakash, A., Koster, J.: Steady thermocapillary convection in a GaAS melt encapsulated by molten $\mathrm{B}_{2} \mathrm{O}_{3}$. J. Mat. Syn. Proc. 4, 43-50 (1996)

11. Priede, J., Gerbeth, G.: Convective, absolute, and global instabilities of thermocapillary-buoyancy convection in extended layers. Phys. Rev. E 56, 4187-4199 (1997)

12. Riley, R.J., Neitzel, G.P.: Instability of thermocapillary-buoyancy convection in shallow layers. Part 1. Characterization of steady and oscillatory instabilities. J. Fluid Mech. 359, 143-164 (1998)

13. Smith, M.: Instability mechanisms in dynamic thermocapillary liquid layers. Phys Fluids 29, 3182-3186 (1986)

14. Smith, M., Davis, S.: Instabilities of dynamic thermocapillary liquid layers. Part 1. Convective instabilities. J. Fluid Mech. 132, 145-162 (1983) 